Check for updates

Cite this: Chem. Commun., 2021, 57, 7693

Received 31st March 2021 Accepted 5th July 2021

DOI: $10.1039 / d 1 c c 01717 a$

rsc.li/chemcomm

\section{Tuning extreme anisotropic thermal expansion in 1D coordination polymers through metal selection and solid solutions $\dagger$}

\author{
Lisa M. van Wyk, (D) Leigh Loots (D) and Leonard J. Barbour (D) *
}

\begin{abstract}
The thermal expansion behaviour of a series of 1D coordination polymers has been investigated. Variation of the metal centre allows tuning of the thermal expansion behaviour from colossal positive volumetric to extreme anomalous thermal expansion. Preparation of solid solutions increased the magnitude of the anomalous thermal expansion further, producing two species displaying supercolossal anisotropic thermal expansion $\left(\mathrm{ZnCoCP}{ }^{\mathrm{HT}} \alpha_{Y 2}=-712 \mathrm{MK}^{-1}, \alpha_{Y 3}=1632 \mathrm{MK}^{-1}\right.$ and $\mathrm{ZnCdCP}^{\mathrm{HT}} \alpha_{Y 2}=-711 \mathrm{MK}^{-1}, \alpha_{Y 3}=1216 \mathrm{MK}^{-1}$ ).
\end{abstract}

Owing to increased thermal motion of their constituent atoms, solids usually expand upon heating; ${ }^{1-3}$ this is known as positive thermal expansion (PTE). ${ }^{1,3,4}$ However, some materials display anomalous thermal expansion behaviour, including negative and zero thermal expansion (NTE and ZTE, respectively), exceptional magnitudes of PTE, as well as extreme anisotropy. Anisotropic thermal expansion is characterised by significantly different linear thermal expansion coefficients for the individual principal axes. ${ }^{1}$ Although anisotropy is expected for materials that do not possess cubic symmetry, extreme anisotropy is uncommon. There have been relatively few reports of anomalous thermal expansion behaviour, ${ }^{4-7}$ but such materials are of great interest in materials science owing to possible applications in optical devices, semiconductor materials and space exploration vehicles. ${ }^{1,2,8,9}$ Furthermore, controlled tuning of the thermal expansion behaviour of solids is desirable for tailoring materials to specific applications. ${ }^{1,7,8,10}$

Previous studies on thermal expansion of crystalline solids have explored molecular crystals, co-crystals and mineral-type compounds. ${ }^{3,11-13}$ However, recent interest in extended materials such as metal-organic frameworks, coordination polymers (CPs), and covalent and hydrogen bonded frameworks have inspired new avenues of investigation. ${ }^{10,14-16}$ Flexible one

Department of Chemistry and Polymer Science, University of Stellenbosch, Matieland 7602, South Africa. E-mail: ljb@sun.ac.za

$\dagger$ Electronic supplementary information (ESI) available: Synthetic procedure, TGA, additional figures, SCXRD, PXRD, DSC and PAS results. CCDC 1940578, 2036084-2036096. For ESI and crystallographic data in CIF or other electronic format see DOI: $10.1039 / \mathrm{d} 1 \mathrm{cc} 01717 \mathrm{a}$ dimensional CPs have received little attention in this regard, but may prove to be excellent candidates for studies of solidstate dynamics.

Recent studies have shown that compounds with "winerack" or "lattice-fence" solid-state packing often exhibit anomalous thermal expansion (Fig. $1 \mathrm{a}-\mathrm{c})^{3,5,7,10,17}$ as a consequence of hinging and twisting around the metal nodes. ${ }^{3,5,10}$ We have also seen that solid solutions show promise for tuning thermal expansion. ${ }^{7,9,18}$ Here we describe the thermal expansion behaviour of a series of isotypical ${ }^{19}$ CPs and their solid solutions, which all employ packing reminiscent of ideal "wine-rack" or "lattice-fence" motifs (Fig. 1d).

Four CPs with the general formula $[\mathrm{M}(\mathrm{bpdc})(\mathrm{bpy}) \cdot 2 \mathrm{DMF}]_{n}$ (MCP, M = Zn, Ni, Co and $\mathrm{Cd}$ ) were prepared solvothermally from a solution of metal nitrate, 4,4'-biphenyldicarboxylic acid (bpdc) and 2,2'-bipyridine (bpy) in $N, N$-dimethylformamide a)

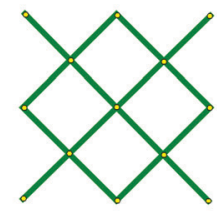

c)

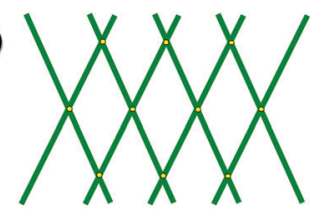

e)

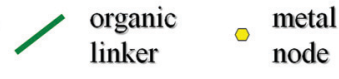

b)

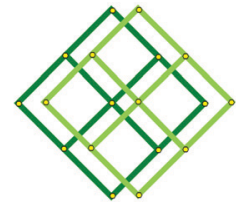

d)

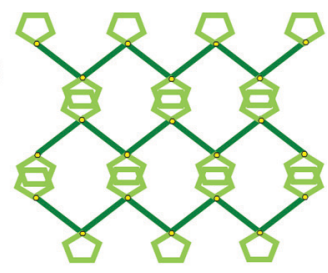

chelating ligand
Fig. 1 Simplified packing motifs commonly associated with anomalous thermal expansion behaviour, including (a) wine-rack, (b) interpenetrated/ offset-layered square-lattice and (c) lattice-fence packing. A simplified depiction of the similar packing motif generated by the 1D materials in this study is shown in (d). Note that, although it appears that the 1D strands in (d) run parallel to the plane of the page, they traverse it. 
(DMF). ZnCP was the first of these to be characterised structurally, but the low-temperature $(100 \mathrm{~K})$ single-crystal X-ray diffraction (SCXRD) data were of poorer quality than we had expected (Fig. S8, ESI $\dagger$ ). SCXRD at $270 \mathrm{~K}$ (the crystals lose solvent at room temperature) yielded better data (Fig. S8, ESI $\dagger$ ), but notably different unit-cell parameters relative to those at $100 \mathrm{~K}$. Differential scanning calorimetry (DSC) revealed that this discrepancy is due to a subtle phase transition, which occurs with an onset temperature of 250-240 K upon cooling (Fig. S17, ESI $\dagger$ ). Comparison of the high- and low-temperature crystal structures of $\mathbf{Z n C P}\left(\mathbf{Z n C P}{ }^{\mathbf{H T}}\right.$ and $\mathbf{Z n C P}{ }^{\mathbf{L T}}$, respectively) reveals that the space group $\left(P 2_{1} / n\right)$ remains unchanged and that, although highly reminiscent of one another, their corresponding unit-cell parameters differ by more than can be expected for normal thermal expansion (Table S1, ESI $\dagger$ ). Nevertheless, the two crystal structures can be considered isotypical. $^{19}$

$\mathbf{Z n C P}^{\mathbf{H T}}$ is comprised of infinite 1D "zigzag" chains $\left(\angle \mathrm{Zn} \cdots \mathrm{Zn} \cdots \mathrm{Zn}=125.34(3)^{\circ}\right)$ that propagate along [602]. Successive metal nodes are linked by means of chelating bpdc ligands (disordered over two positions with site occupancies of 0.53 and 0.47 ). Each metal centre is also bound to a chelating bpy ligand (disordered over two positions with site occupancies of 0.67 and 0.33 ), resulting in an overall highly distorted octahedral coordination environment. The 1D strands interdigitate via offset $\pi \cdots \pi$ interactions between bpy moieties of adjacent CP strands. This arrangement produces 1D guestaccessible channels that propagate along [100] and that contain DMF guest molecules (Fig. 2 and Fig. S10, ESI $\dagger$ ). We have explored the transient porosity ${ }^{20}$ of $\mathbf{Z n C P}$ as part of a separate study, ${ }^{21}$ and a view along the $1 \mathrm{D}$ channels serves as a convenient frame of reference with respect to comparing the crystal structures.

The phase transition from $\mathbf{Z n C P} \mathbf{P}^{\mathbf{H T}}$ to $\mathbf{Z n C P} \mathbf{P}^{\mathbf{L T}}$ (based on the crystal structures determined at 270 and $100 \mathrm{~K}$, respectively) involves a relatively minor structural rearrangement. The connectivity of $\mathbf{Z n C P} \mathbf{P}^{\mathbf{H T}}$ is maintained in $\mathbf{Z n C P} \mathbf{P}^{\mathbf{L T}}$, and the disorder of the ligands remains similar (50:50 and 62:38 for bpdc and bpy, respectively). However, after the phase change the zigzag angle is markedly different. These changes lead to slight distortion of the 1D channel, with lengthening along [010] and shortening along [001] (Fig. 2 and Fig. S9 and S14, ESI $\dagger$ ).

The phase change experienced by ZnCP upon cooling is due to an abrupt decrease in the $\mathbf{M} \cdots \mathbf{M} \cdots \mathbf{M}$ zigzag angle from $123.89(3)^{\circ}$ at $250 \mathrm{~K}$ to $121.66(3)^{\circ}$ at $240 \mathrm{~K}$ (Fig. S16 and Table $\mathrm{S} 13, \mathrm{ESI} \dagger)$. This is accompanied by elongation of the channels along [010], with concomitant narrowing along the vector perpendicular to (001) ( $c f$. Fig. 2 and Fig. S8, ESI $\dagger$ ). These changes are manifested as elongation of the crystallographic $a$ and $b$ axes, a small increase in the $\beta$ angle, and shortening of the $c$ axis (Fig. S13, ESI $\dagger$ ).

Determination of unit-cell parameters at $10 \mathrm{~K}$ intervals from 270 to $100 \mathrm{~K}$ shows that both phases of ZnCP undergo dramatic anisotropic thermal expansion (Fig. S14, ESI $\dagger$ ). Respectively, the linear thermal expansion coefficients ${ }^{22} \alpha_{y 1}, \alpha_{y 2}$ and $\alpha_{y 3}$ for $\mathbf{Z n C P}^{\mathbf{H T}}$ are $100,-462$ and $882 \mathbf{M K}^{-1}$, while those for $\mathbf{Z n C P}{ }^{\mathbf{L T}}$ are $69,-57$ and $275 \mathrm{MK}^{-1}$. The matrices relating the principal axes to the unit-cell axes are given in Fig. S25, S26 and S39 of the ESI. $\dagger$ Taken together, the linear thermal expansion coefficients of $\mathbf{Z n C P}^{\mathbf{H T}}$ represent some of the most extreme anisotropic values recorded to date for any known crystalline materials (Table S17, ESI $\dagger$ ). Moreover, both phases also undergo colossal $\left(\alpha>100 \mathrm{MK}^{-1}\right)$ volumetric PTE $\left(\alpha_{\mathrm{V}}=575\right.$ and $292 \mathrm{MK}^{-1}$, respectively). Since the $1 \mathrm{D}$ channels propagate along [100] (Fig. 2), we can infer that most of the thermal expansion anisotropy is due to distortion of the host around the channels. In $\mathbf{Z n C P} \mathbf{P}^{\mathbf{H T}}$ the expansion coefficient approximately along [100] (i.e., $\left.\alpha_{Y 1}\right)$ is relatively modest, while that along [010] (i.e., $\alpha_{Y 2}$ ) is highly negative. The expansion coefficient normal to (001) is very highly positive in both cases. These observations
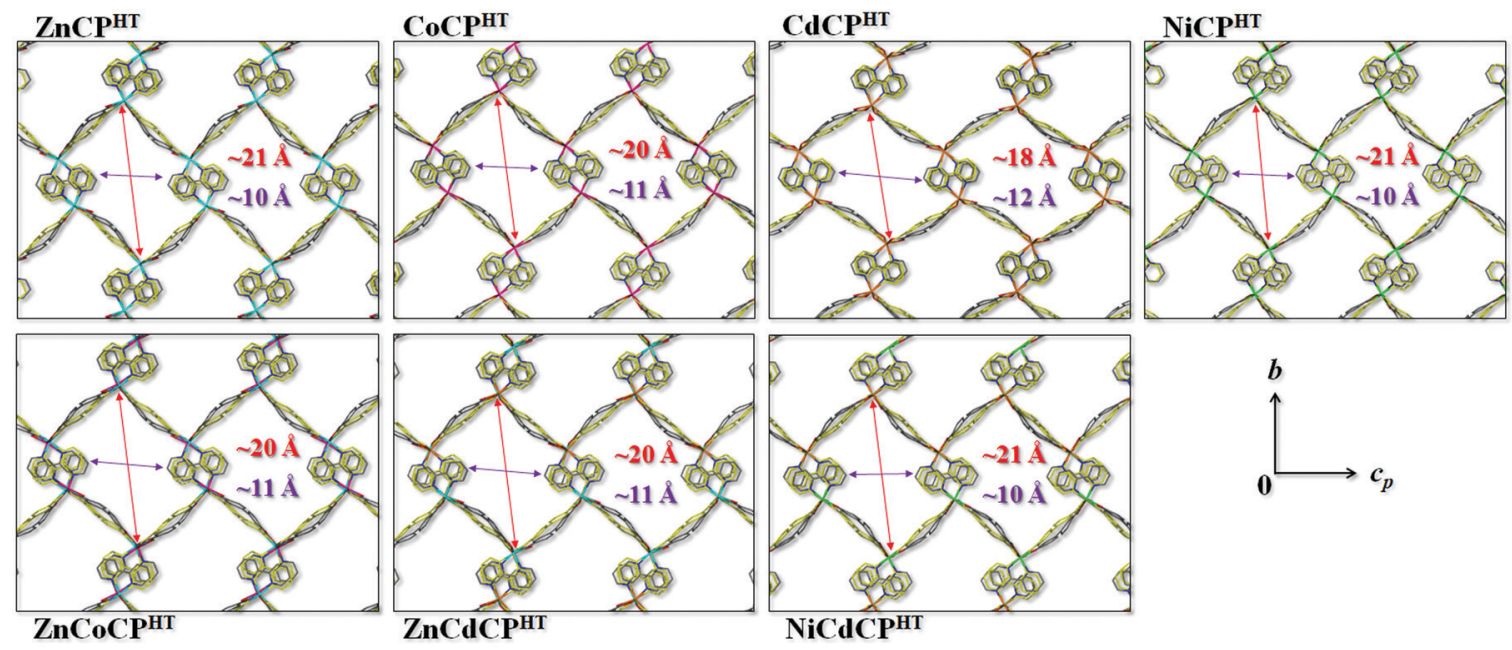

Fig. 2 Packing of the high-temperature phases (270 K) of all CPs described in this study, viewed along [100]. In all cases both ligands are disordered over two positions. The minor component of disorder for each ligand is shown in yellow. The approximate atom-to-atom dimensions are indicated for each CP. Both metal atoms are shown in the representations of the solid solutions, but may be superimposed. Guest molecules and hydrogen atoms have been omitted for clarity. Note that the red arrow runs approximately along the $b$ axis and the purple arrow approximately along the $c$ axis 
comport with gradual narrowing (see ESI $\dagger$ ) of the zigzag angle with decreasing temperature, as also occurs abruptly during the phase transition.

Previously we $\mathrm{e}^{23,24}$ and others ${ }^{3,6,25-27}$ were successful in tuning the thermal expansion behaviour of structural analogues by modulating their composition. In this context we explored metal node substitution for facile modification of the coordination geometries about various metal centres; a series of MCP analogues was prepared with $\mathrm{M}=\mathrm{Ni}$, Co and Cd. Using SCXRD, we established that all of these materials are isotypes ${ }^{19}$ of $\mathbf{Z n C P}$ (Fig. 2 and Table S1, ESI $\dagger$ ). They also undergo subtle low temperature phase transitions in the ranges 250-240, 260-250 and 200-190 K, respectively (Fig. S14, ESI $\dagger$ ).

Multiple attempts were made to determine successive crystal structures across the phase change with slow cooling. However, in all cases the crystals fractured during the transition, thus yielding poor quality diffraction data from which it was only possible to determine unit cell parameters, the space group and estimates of $\mathbf{M} \cdots \mathbf{M} \cdots \mathbf{M}$ angles (with the exception of CoCP).

Upon cooling, the changes in the unit-cell parameters of NiCP across the phase change are similar to those of ZnCP, with expansion along [010] and concomitant contraction along [001]. Moreover, the $\mathbf{M} \cdots \mathbf{M} \cdots \mathbf{M}$ zigzag angle in NiCP also narrows across the phase change (from $123.31(3)^{\circ}$ at $250 \mathrm{~K}$ to $116.38(3)^{\circ}$ at $240 \mathrm{~K}$, Table S13 and Fig. S16b, ESI $\left.\dagger\right)$. Interestingly, in the cases of CoCP and CdCP, cooling across the phasechange results in shortening of the $b$ axis and narrowing of the $\beta$ angle, with elongation of the $c$ axis (Fig. S14, ESI $\dagger$ ). Therefore, with regard to these parameters, the phase changes in CoCP and $\mathbf{C d C P}$ are inversely related to those of $\mathbf{Z n C P}$ and NiCP. This is also reflected in the increase of the $\mathbf{M} \cdots \mathbf{M} \cdots \mathbf{M}$ zigzag angle in CdCP from $140.24(1)^{\circ}$ at $200 \mathrm{~K}$ to $145.29(1)^{\circ}$ at $190 \mathrm{~K}$ (Table S13 and Fig. S16, ESI $\dagger$ ). The phase change temperatures for CoCP and CdCP are significantly different (ca. 260 and $200 \mathrm{~K}$, respectively).

Variable-temperature unit-cell determinations for $\mathbf{C d C P}$ were carried out as for ZnCP. Owing to facile desolvation and relatively high phase-change temperatures of $\mathbf{C o C P}^{\mathbf{H T}}$ and $\mathbf{N i C P}^{\mathbf{H T}}$ (both ca. $250 \mathrm{~K}$ ), an environmental solvent cell was used for unit cell determinations in the range 280 to $310 \mathrm{~K}$ (see ESI $\dagger$ ). NiCP ${ }^{\text {HT }}$ exhibits colossal linear PTE along $Y 2$ and $Y 3$, while CoCP $^{\text {HT }}$ exhibits linear NTE along Y3 (Fig. 3 and Fig. S27, Table S14, ESI $\dagger)$. Both exhibit colossal volumetric PTE $\left(\alpha_{\mathrm{V}}=371 \mathrm{MK}^{-1}\right.$ for $\mathbf{N i C P}^{\mathbf{H T}}$ and $\left.239 \mathbf{M K}^{-1} \mathbf{C o C P}^{\mathbf{H T}}\right)$. In $\mathbf{C o C P}^{\mathbf{H T}}$ both linear PTE and NTE occur in the $a c$ plane between adjacent 1D strands. The magnitudes of thermal expansion (both linear and volumetric) of $\mathbf{N i C P}^{\mathbf{H T}}$ and $\mathbf{C O C P}^{\mathbf{H T}}$ are substantially lower than that of ZnCP. Both phases of CdCP exhibit extreme anisotropic thermal expansion (Fig. 3 and Fig. S40, Table S16, ESI $\dagger$ ), similar to $\mathbf{Z n C P}\left(\mathbf{C d C P}^{\mathbf{H T}} \alpha_{Y 2}=778 \mathrm{MK}^{-1}, \alpha_{Y 3}=-389 \mathrm{MK}^{-1}\right.$ and $\left.\alpha_{\mathrm{V}}=531 \mathrm{MK}^{-1}\right)$.

Tuning of thermal expansion behaviour in metal containing materials may also be achieved by the preparation of solid solutions. $^{7-9,18,28}$ Three solid solutions were prepared by the addition of equimolar amounts of two metal nitrates to the crystallisation solution $\left(\mathbf{M M}^{\prime} \mathbf{C P}, \mathbf{M M}^{\prime}=\mathrm{ZnCo}, \mathrm{ZnCd}, \mathrm{NiCd}\right)$. However, energy dispersive X-ray analysis showed that each of

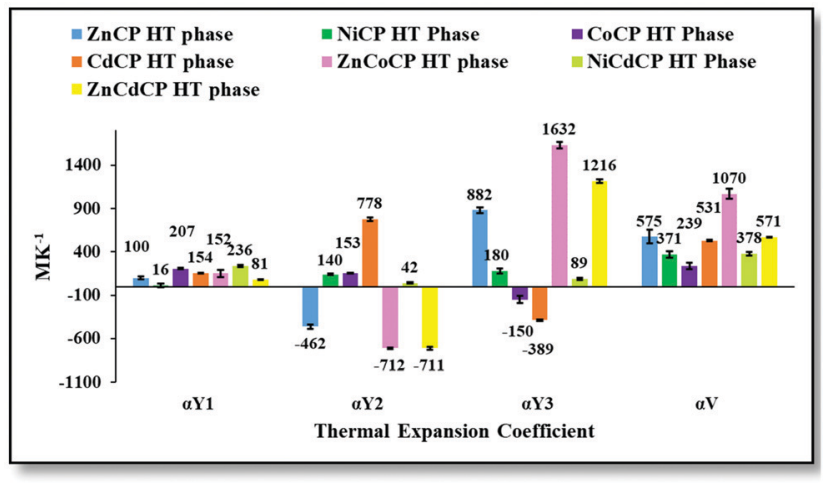

Fig. 3 Thermal expansion coefficients for the high-temperature phases of the CPs. Error bars and specific values are indicated.

the resulting materials contains substantially different amounts of metal ions (Table S18, ESI $\dagger$ ). All of the solid solutions have similar packing arrangements and phase change behaviour to those observed for ZnCP. As expected, the structure of $\mathbf{Z n C o C P}\left(\left[\mathrm{Zn}_{0.92} \mathrm{Co}_{0.08}(\mathrm{bpdc})(\mathrm{bpy}) \cdot 2 \mathrm{DMF}\right]_{n}\right)$ and $\mathbf{Z n C d C P}$ $\left(\left[\mathrm{Zn}_{0.92} \mathrm{Cd}_{0.08}(\mathrm{bpdc})(\mathrm{bpy}) \cdot 2 \mathrm{DMF}\right]_{n}\right)$ are most similar to that of ZnCP; the packing is likely directed by the major metal component. This holds true for both the HT and LT phases of these materials. The structure of $\mathbf{N i C d C P}{ }^{\mathbf{H T}}\left(\left[\mathrm{Ni}_{0.83} \mathrm{Cd}_{0.17}\right.\right.$ (bpdc)(bpy). $2 \mathrm{DMF}]_{n}$ ) is also closer to that of $\mathbf{N i C P}^{\mathbf{H T}}$. However, owing to the notable difference in packing between $\mathbf{N i C P}^{\mathbf{L T}}$ and $\mathbf{C d C P}^{\mathbf{L T}}$ (Fig. S9, ESI $\dagger$ ), NiCdCP ${ }^{\mathbf{L T}}$ adopts an intermediate packing arrangement, which is similar to that of the other solid solutions.

Variable-temperature unit-cell determinations were carried out to determine the thermal behaviour of the solid solutions. With regard to changes in their unit-cell parameters, all three solid solutions exhibit phase-change behaviour similar to that of $\mathbf{Z n C P}$, albeit at different onset temperatures. $\mathbf{Z n C o C P} \mathbf{H T}^{\mathbf{H T}}$ exhibits colossal linear NTE along $Y 2\left(\alpha_{Y 2}=-712 \mathrm{MK}^{-1}\right)$ and supercolossal (an order of magnitude larger than colossal) ${ }^{29}$ PTE along $Y 3\left(\alpha_{Y 3}=1632 \mathrm{MK}^{-1}\right)$. $\mathbf{Z n C o C P}^{\mathbf{H T}}$ also displays supercolossal volumetric PTE $\left(\alpha_{\mathrm{V}}=1070 \mathrm{MK}^{-1}\right.$, Fig. 3 and Table S16, ESI $\dagger$ ). Both $\alpha_{\mathrm{V}}$ and the degree of anisotropy are far greater for $\mathbf{Z n C o C P}{ }^{\mathbf{H T}}$ than for $\mathbf{Z n C P}^{\mathbf{H T}}$ or $\mathbf{C o C P}^{\mathbf{H T}}$, and both $Y 2$ and $Y 3$ are nearly twice those for $\mathbf{Z n C P} \mathbf{P}^{\mathbf{H T}}$ and many times larger than for $\mathbf{C o C P} \mathbf{P}^{\mathbf{H T}}$. Interestingly, the thermal expansion behaviour of $\mathbf{Z n C o C P} \mathbf{P}^{\mathbf{L T}}$ is more similar to that of $\mathbf{C o C P}^{\mathbf{L T}}$; it undergoes linear ZTE along $Y 2$ and PTE along the other principal axes (Table S16 and Fig. S40, ESI $\dagger$ ), resulting in colossal volumetric PTE.

The thermal expansion of $\mathbf{Z n C d C P} \mathbf{P}^{\mathbf{H T}}$ is analogous to that of the $\mathbf{Z n C o C P}^{\mathbf{H T}}$ (Fig. 3 and Table S16, ESI $\dagger$ ). Similarly, to ZnCoCP $^{\text {HT }}$ the linear NTE along $Y 2$ is colossal and the PTE along $Y 3$ is supercolossal $\left(\alpha_{Y 2}=-711 \mathrm{MK}^{-1}, \alpha_{Y 3}=1216 \mathrm{MK}^{-1}\right)$. However, its volumetric PTE is colossal $\left(\alpha_{\mathrm{V}}=571 \mathrm{MK}^{-1}\right)$. The orientations of the principal axes and thus the mechanism for thermal expansion in $\mathbf{Z n C d C P} \mathbf{P}^{\mathbf{H T}}$ are analogous to those of ZnCoCP $^{\text {HT }}$ (Fig. S39, ESI $\dagger$ ). The linear thermal expansion behaviour of NiCdCP${ }^{\mathbf{H T}}$ differs from that of both $\mathbf{N i C P}^{\mathbf{H T}}$ and $\mathbf{C d C P}^{\mathbf{H T}}$ (Fig. 3); the magnitudes of the coefficients along Y2 
Table 1 Examples of extreme anisotropic thermal expansion

\begin{tabular}{|c|c|c|c|c|c|}
\hline Material & $\alpha_{Y 1}\left(\mathrm{MK}^{-1}\right)$ & $\alpha_{Y 2}\left(\mathrm{MK}^{-1}\right)$ & $\alpha_{Y 3}\left(\mathrm{MK}^{-1}\right)$ & $\alpha_{V}\left(\mathrm{MK}^{-1}\right)$ & Ref. \\
\hline CA-Pyz & -1375 & 196 & 1524 & 245 & 29 \\
\hline$\left[\mathrm{Zn}_{2}\left(\mathrm{fu}-\mathrm{L}^{1}\right)_{2} \text { dabco }\right]_{n}$ & -380 & 1161 & 14.6 & 837 & 6 \\
\hline$\left[\mathrm{Fe}_{0.84} \mathrm{Ni}_{0.16}(\mathrm{bpac})\left(\mathrm{Au}(\mathrm{CN})_{2}\right)_{2}\right] \cdot 2 \mathrm{EtOH}$ & -3200 & 5200 & 1500 & 3200 & 7 \\
\hline$\left(\mathrm{Mn}_{0.95} \mathrm{Ni}_{0.05}\right) \mathrm{CoGe}$ & -1804 & 1265 & 46 & -624 & 18 \\
\hline $\mathrm{ZnCoCP}^{\mathrm{HT}}$ & 152 & -712 & 1632 & 1070 & This work \\
\hline $\mathrm{ZnCdCP}^{\mathrm{HT}}$ & 81 & -711 & 1216 & 570 & This work \\
\hline
\end{tabular}

and $Y 3$ for $\mathbf{N i C d C P} \mathbf{P}^{\mathbf{H T}}$ are notably smaller than those for $\mathbf{N i C P} \mathbf{P}^{\mathbf{H T}}$ and $\mathrm{CdCP}^{\mathrm{HT}}$.

During the course of this study we noted a discrepancy between the unit-cell parameters at $100 \mathrm{~K}$ obtained after incremental cooling (periodic unit-cell determinations) and flashcooling the crystal (rapid cooling from ambient to $100 \mathrm{~K}$ )-see Tables S3-S9, S14 (ESI $\dagger$ ). The thermal expansion behaviour appears to be attenuated when the crystal is flash-cooled. This observation was further investigated by means of differential scanning calorimetry at different cooling rates and in triplicate, which supports the assertion that higher cooling rates lead to attenuation of the phase-change (see ESI $\dagger$ ).

All of the CPs display interesting thermal expansion behaviour, ranging from colossal volumetric PTE to supercolossal linear thermal expansion. Their thermal expansion coefficients are comparable to the highest reported values for anisotropic thermal expansion (Table 1 and Table S17, ESI $\dagger$ ). An overview of reports of colossal (and supercolossal) anisotropic thermal expansion is presented in Table 1 and a more comprehensive survey of the literature is given in Table S17 (ESI $\dagger$ ). Almost all of the materials in this study exceed reports of both linear and volumetric thermal expansion in 1D CPs (Table S17, ESI $\dagger$ ). The only material exceeding (dramatically) the values reported here requires a spin-crossover transition to effect exceptional thermal expansion of a $2 \mathrm{D}$ framework. ${ }^{7}$ It is interesting to note that the best performing materials in this study $\left(\mathbf{Z n C o C P}^{\mathbf{H T}}\right.$ and $\mathbf{Z n C d C P}^{\mathbf{H T}}$ ) and two of the four best performing materials in the literature are all solid solutions. Therefore, solid solutions should be considered a viable method of increasing the thermal expansion properties of a given class of material.

We thank the National Research Foundation (NRF) of South Africa for financial support and the Central Analytical Facilities at Stellenbosch University for EDX analyses.

\section{Conflicts of interest}

There are no conflicts to declare.

\section{Notes and references}

1 J. Chen, L. Hu, J. Deng and X. Xing, Chem. Soc. Rev., 2015, 44, 3522-3567.

2 K. Takenaka, Sci. Technol. Adv. Mater., 2012, 13, 013001.
3 Z. Liu, Q. Gao, J. Chen, J. Deng, K. Lin and X. Xing, Chem. Commun., 2018, 54, 5164-5176.

4 D. Das, T. Jacobs and L. J. Barbour, Nat. Mater., 2010, 9, 36-39.

5 W. Cai and A. Katrusiak, Nat. Commun., 2014, 5, 4337.

6 S. Henke, A. Schneemann and R. A. Fischer, Adv. Funct. Mater., 2013, 23, 5990-5996.

7 B. R. Mullaney, L. Goux-Capes, D. J. Price, G. Chastanet, J.-F. Létard and C. J. Kepert, Nat. Commun., 2017, 8, 1053.

$8 \mathrm{~J}$. Chen, Q. Gao, A. Sanson, X. Jiang, Q. Huang, A. Carnera, C. G. Rodriguez, L. Olivi, L. Wang, L. Hu, K. Lin, Y. Ren, Z. Lin, C. Wang, L. Gu, J. Deng, J. P. Attfield and X. Xing, Nat. Commun., $2017,8,14441$.

9 H. Yamamoto, T. Imai, Y. Sakai and M. Azuma, Angew. Chem., Int. Ed., 2018, 57, 8170-8173.

10 L. D. Devries, P. M. Barron, E. P. Hurley, C. Hu and W. Choe, J. Am. Chem. Soc., 2011, 133, 14848-14851.

11 T. A. Mary, J. S. Evans, T. Vogt and A. W. Sleight, Science, 1996, 272, 90-92.

12 J. S. O. Evans, Z. Hu, J. D. Jorgensen, D. N. Argyriou, S. Short and A. W. Sleight, Science, 1997, 275, 61-65.

13 A. L. Goodwin, M. Calleja, M. J. Conterio, M. T. Dove, J. S. O. Evans, D. A. Keen, L. Peters and M. G. Tucker, Science, 2008, 319, 794-797.

14 J. P. Zhang, P. Q. Liao, H. L. Zhou, R. B. Lin and X. M. Chen, Chem. Soc. Rev., 2014, 43, 5789-5814.

15 Z. Chang, D. H. Yang, J. Xu, T. L. Hu and X. H. Bu, Adv. Mater., 2015, 27, 5432-5441.

16 S. R. Batten, N. R. Champness, X. Chen, J. Garcia-Martinez, S. Kitagawa, L. Ohrstrom, M. O'Keeffe, M. Paik Suh and J. Reedijk, CrystEngComm, 2012, 14, 3001-3004.

17 S. J. Hunt, M. J. Cliffe, J. A. Hill, A. B. Cairns, N. P. Funnell and A. L. Goodwin, CrystEngComm, 2015, 17, 361-369.

18 Q. Ren, W. Hutchison, J. Wang, A. Studer, G. Wang, H. Zhou, J. Ma and S. J. Campbell, ACS Appl. Mater. Interfaces, 2019, 11, 17531-17538.

19 L. J. Barbour, D. Das, T. Jacobs, G. O. Lloyd and V. J. Smith, in Supramolecular Chemistry: from Molecules to Nanomaterials, ed. P. A. Gale and J. W. Steed, John Wiley \& Sons, Ltd., Hoboken, 2012.

20 L. J. Barbour, Chem. Commun., 2006, 1163.

21 L. M. van Wyk and L. J. Barbour, Cryst. Growth Des., 2021, 21, 3056-3062.

22 M. J. Cliffe and A. L. Goodwin, J. Appl. Crystallogr., 2012, 45, $1321-1329$

23 I. Grobler, V. J. Smith, P. M. Bhatt, S. A. Herbert and L. J. Barbour, J. Am. Chem. Soc., 2013, 135, 6411-6414.

24 E. R. Engel, V. J. Smith, C. X. Bezuidenhout and L. J. Barbour, Chem. Mater., 2016, 28, 5073-5079.

25 L. Hu, J. Chen, J. Xu, N. Wang, F. Han, Y. Ren, Z. Pan, Y. Rong, R. Huang, J. Deng, L. Li and X. Xing, J. Am. Chem. Soc., 2016, 138, 14530-14533.

26 J. S. Ovens and D. B. Leznoff, Inorg. Chem., 2017, 56, 7332-7343.

27 H. L. Zhou, R. B. Lin, C. T. He, Y. B. Zhang, N. Feng, Q. Wang, F. Deng, J. P. Zhang and X. M. Chen, Nat. Commun., 2013, 4, 1-8.

28 H.-L. Zhou, J.-P. Zhang and X.-M. Chen, Front. Chem., 2018, 6, 1-7.

29 H. Liu, M. J. Gutmann, H. T. Stokes, B. J. Campbell, I. R. Evans and J. S. O. Evans, Chem. Mater., 2019, 31, 4514-4523. 